\title{
THE DISTRIBUTION OF SURFACE STRAIN IN THE CADAVERIC LUMBAR SPINE
}

\author{
J. S. SHAH, W. G. J. HAMPSON, M. I. V. JAYSON \\ From the Departments of Physics and of Medicine, University of Bristol
}

\begin{abstract}
The fourth lumbar vertebrae and L4-5 discs from six cadaveric lumbar spines were subjected to detailed strain gauge analysis under conditions of controlled loading. With central compression loads, maximal compressive strain was found to occur near the bases of the pedicles and on both superficial and deep surfaces of the pars interarticularis, which emphasises the importance of the posterior elements of lumbar vertebrae in transmitting load. Radial bulge and tangential strain of the disc wall were maximal at the posterolateral surface, in agreement with the fact that disc degeneration and prolapse commonly occur there. Under posterior offset loads simulating extension, both compressive and tensile strains were found to be increased on both surfaces of the pars interarticularis, which suggests that hyperextension may lead to stress fractures and spondylolisthesis. Posterior offset loads also increased the radial bulge of the posterior disc wall and tangential strain at the anterior surface of the disc. Anterior offset loads simulating flexion increased the radial bulge of the anterior disc wall and tangential strain at the posterior surface of the disc. These findings are compatible with movement of the nucleus pulposus within the disc during flexion and extension. This hypothesis was supported by post-mortem discography.
\end{abstract}

A number of workers have measured the expansion of the intervertebral disc in the isolated lumbar spine under axial load, whereas others have determined the ultimate loads required to produce failure of the vertebrae and discs. Previous studies, however, have not measured the relative distribution of surface strain in lumbar vertebrae under loading conditions that simulate everyday activities, nor provided information on strain in relation to the behaviour of the disc. The aim of this investigation was to correct these deficiencies.

\section{METHODS AND MATERIALS}

The lumbar spines were removed intact from previously healthy adults who had died shortly after road accidents but with no evidence of spinal injury. Anteroposterior and lateral radiographs were taken, together with discography using 40 per cent Hypaque in order to ensure that only undamaged spines with normal discs were used. The six selected spines were stored in sealed polythene bags at -25 degrees Celsius. To prepare for testing, the specimens were thawed and the muscles were removed with careful preservation of the ligaments and discs. Horizontal saw cuts were made between the uppermost and middle thirds of the third lumbar vertebrae and the middle and lowermost thirds of the fifth lumbar vertebrae so as to provide specimens as shown in Figure 1.

Seventeen sites on the surfaces of the fourth lumbar vertebrae were chosen for examination, as numbered in Figure 1. They were at the centre of the anterior surface of the body (1); near the anterolateral and posterolateral surfaces of the upper and lower vertebral rims $(2,3,4,5,6,8,9$ and 11$)$; at the bases of pedicles ( 7 and
10); on the posterior surface of the body (12 and 13); and on both the superficial and deep surfaces of the pars interarticularis $(14,15,16$ and 17).

At each site the bone was exposed and cleaned with ether. A metal foil strain gauge was then bonded to the surface by cyano-acrylic adhesive. Rosette strain gauges were used, each consisting of three separate measuring elements mounted at intervals of 45 degrees. These enabled the directions and magnitudes of both principal compression and tensile strains to be determined at each site (Lanyon 1974).

In addition, radial bulging of the disc and tangential strain (circumferential stretching) of the disc wall were measured by transducers at six sites on the surface of the L4-5 disc (Figure 1, A-F). The stainless steel arm of the transducer, connected to a curved flexible metal strip bonded with a single metal foil strain gauge, was placed with its tip in contact with the surface of the disc; bulging displaced the arm and deformed the strain gauge. Tangential strain was measured by a transducer fastened at both ends to the surface of the disc with fine steel pins and cyano-acrylic adhesive. Circumferential stretching deformed the single metal foil strain gauge bonded to its surface.

Each instrumented spinal segment was wrapped in tissue moistened with saline to prevent dehydration. It was placed in a specimen holder with interlocking hinges at right angles to each other (Fig. 2) and tested in a Dennison tensometer converted for compression.

Three different modes of loading were used.

Central axial loading. At the start of each experiment a pivoted mounting on the top plate of the specimen holder was adjusted by using appropriate wedges so that the top and the bottom plates were exactly parallel. The pivot was then locked so that the plates could only move towards or away from each other, the interlocking hinges preventing any flexion, extension or rotation of the spine.

J. S. Shah, B.Sc., Ph.D., M.Inst.P., H. O. Wills Physics Laboratory, University of Bristol, Royal Fort, Tindall Avenue, Bristol BS8 1TL. W. G. J. Hampson, M.A., F.R.C.S.(Edin.), Southmead Hospital, Westbury-on-Trym, Bristol BS10 5NB, England.

Professor M. I. V. Jayson, M.D., F.R.C.P., University Centre for the Study of Chronic Rheumatism, University of Manchester, Hope Hospital, Eccles Old Road, Salford M6 8HD, England.

Requests for reprints should be sent to Professor Jayson. 

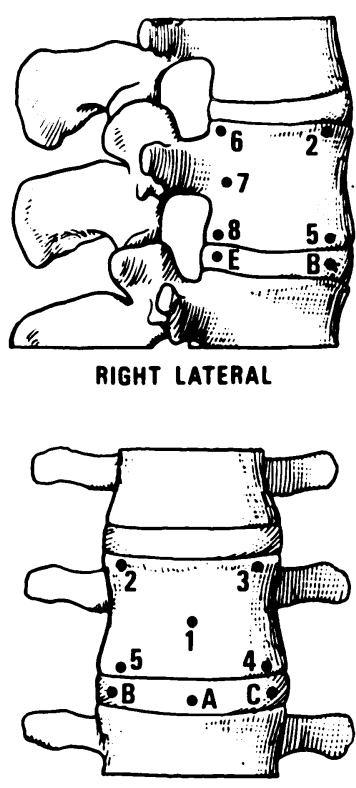

ANTERIOR

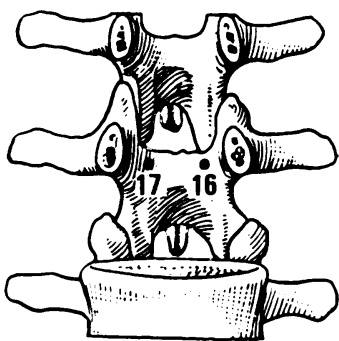

ANTERIOR VIEW OF PARS INTERARTICULARIS

(VERTEBRAL BODIES CUT AWAY)

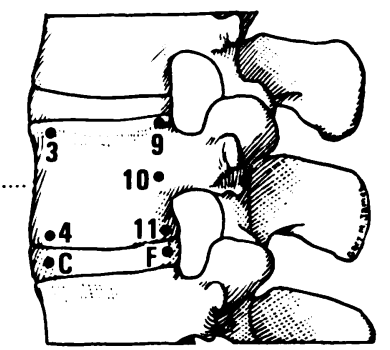

LEFT LATERAL
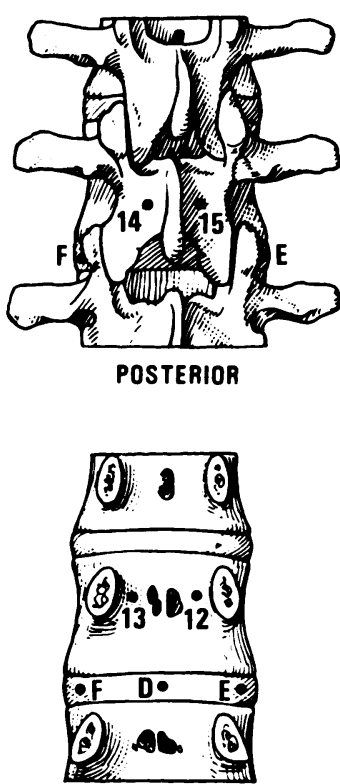

POSTERIOR VIEW OF VERTEBRAL BODIES (PEDICLES \& PARS INTER-

-ARTICULARIS CUT AWAY

Fig. 1

The spinal segment showing the seventeen transducer sites on L4 and 6 on the L4-5 disc.

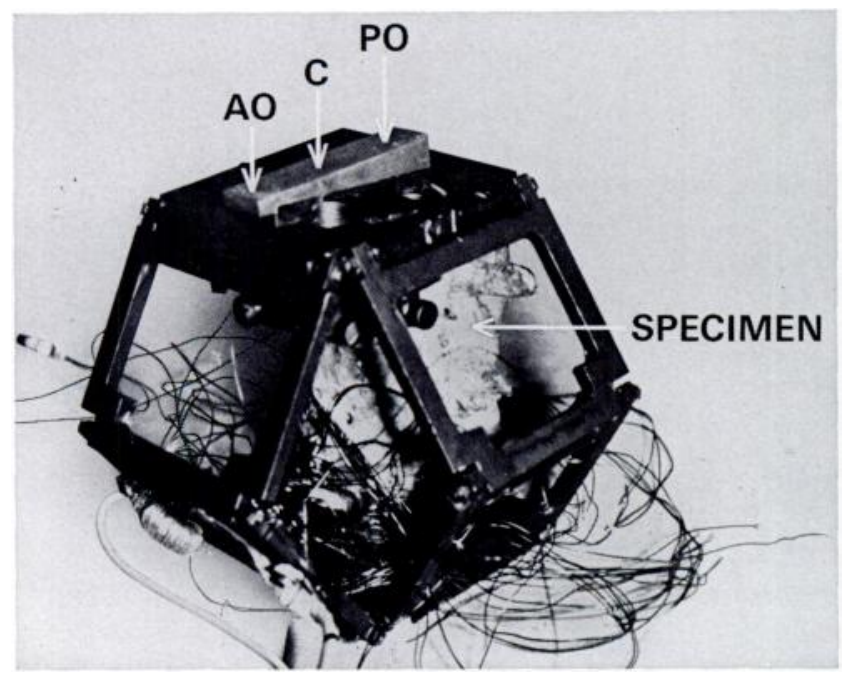

Fig. 2

Specimen in holder showing interlocking hinges and pivotable top plate. Central $(C)$, anterior offset $(A O)$ and posterior offset $(P O)$ loads can be applied.
Posterior offset loading. The compression load was applied in a line parallel to the longitudinal axis of the specimen, but offset posteriorly by three centimetres (Fig. 2). In this way the pivot in the top plate of the specimen holder was freed, allowing anteroposterior flexion and extension of the specimen although the interlocking hinges still prevented lateral flexion and rotation.

Anterior offset loading. The loading mode was identical except that the compression load was applied offset anteriorly by three centimetres (Fig. 2).

In each experiment loads were applied in ten steps up to 192 kilograms (1883.5 Newtons), and strain gauge readings were taken from all seventeen sites on the bone and all six sites on the disc for each load.

The strain gauge signals were measured by a half bridge arrangement using matched dummy gauges to eliminate errors due to temperature drift. The signals were amplified and analysed on a computer-based data logging system shown schematically in Figure 3.

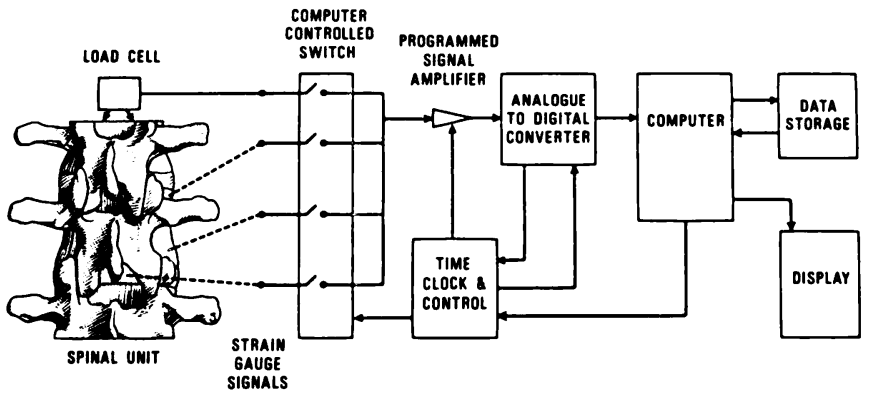

Fig. 3

The scheme for measuring strains in the spinal unit

At each load, the readings were recorded twenty times over a period of fifteen seconds. The computer took the mean and converted it into principal tensile and compression components according to the formula of Dally and Riley (1965). As the system was capable of recording twelve channels, readings from any four rosette strain gauges could be recorded simultaneously.

Throughout the experiments checks were made of previous recordings to ensure that no drift was taking place. The overall accuracy of the measuring system was \pm 0.5 per cent. Faulty bonding of a gauge to bone usually produced obvious errors which were corrected by fresh bonding of the gauge.

\section{RESULTS}

Six spines from four men and two women aged between sixteen and forty-one years were studied. The detailed strain results from all six with central, anterior offset and posterior offset axial loadings were recorded and may be obtained by writing to the authors. Comparable figures are available for all the different applied loads. Typical results are presented to illustrate the main findings of our investigation.

Figure 4 shows an example of the principal compression and tensile strains recorded during central loading at site 11 on the vertebral body, and radial bulge and tangential strain at site $E$ on the disc of the second lumbar spine tested. Both the principal compression and tensile strains on the bone surface increased in a linear fashion with load, while radial bulge and tangential strain on the disc wall increased but in a non-linear 
fashion. In other words, the disc wall became progressively stiffer with increasing load. Similar patterns of change with load occurred at other bone and disc sites. Figure 5 demonstrates the marked variation in the magnitudes of these measurements at the same sites in all six specimens. This wide variation was typical of those at other sites in both vertebrae and discs. However, the relative strain distributions between the seventeen vertebral sites and six disc sites were consistent in all six spines.

Figure 6 illustrates the principal compression strains at a load of 150 kilograms (1471.5 Newtons) of central axial compression, listing the sites in decreasing order of magnitude. The highest compressive strains were recorded near the bases of the pedicles and on the superficial and deep surfaces of the pars interarticularis. The lowest compressive strains were recorded at the inferior vertebral rims.

Figure 7 shows the principal tensile strains in decreasing order of magnitude at a load of 150 kilograms (1471.5 Newtons) of central axial compressive load. The highest tensile strains occurred at the posterior vertebral rims and the lowest tensile strain at the centre of the anterior surface of the vertebral body.
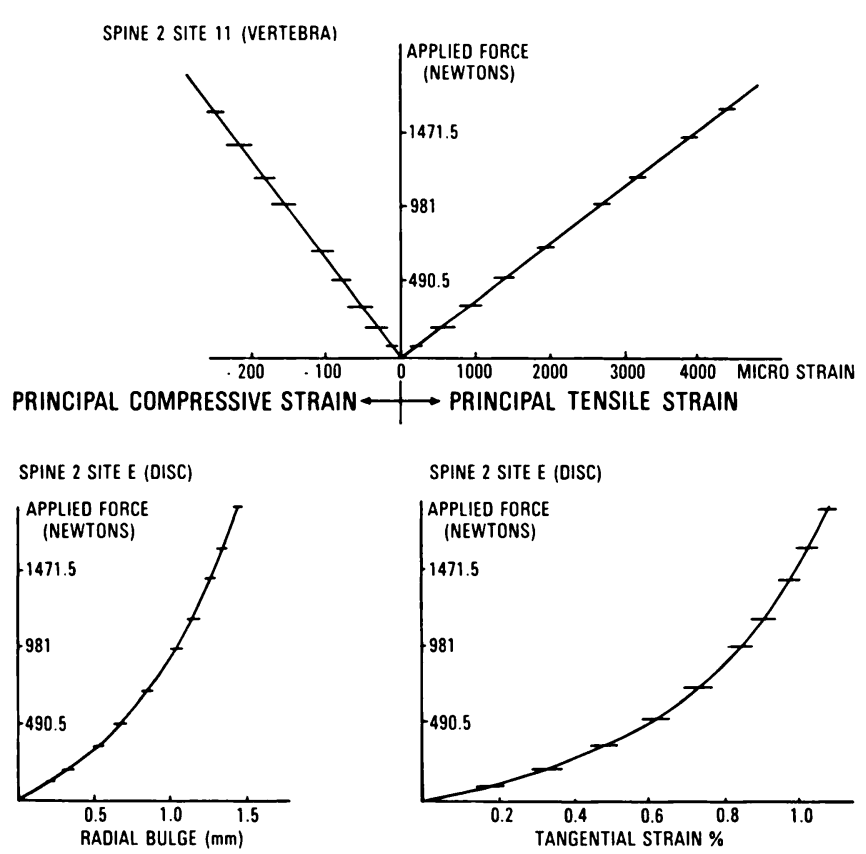

Fig. 4

Typical strain/force and bulge/force patterns at sites 11 and $E$ on the vertebra and disc surfaces. The standard deviations of strain values at each load are shown.

Figure 8 shows the ranges of radial bulges and tangential strains in the L4-5 disc wall at a load of 150 kilograms (1471.5 Newtons) of central axial load. Both were highest at the back of the disc.

The results of the offset loading experiments are summarised in Figure 9. Posterior offset loading increased the compression strain at the bases of the pedicles, and both compression and tensile strains in the pars interarticularis. It also produced a larger bulge of the posterior disc wall, but the tangential strain was maximal at the anterior surface of the disc.

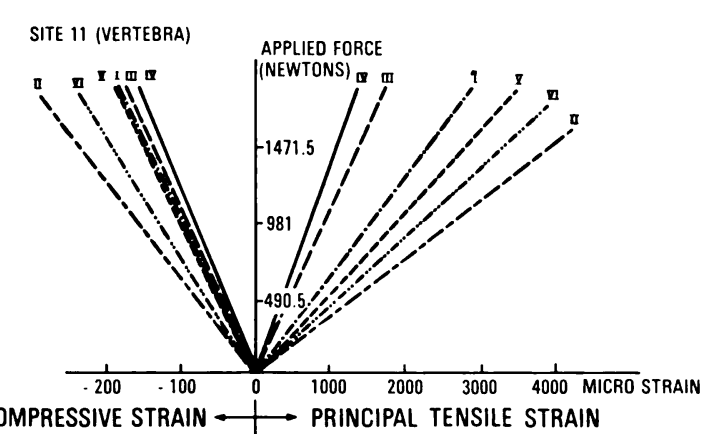
PRINCIPAL COMPRESSIVE STRAIN $\longrightarrow$ PRINCIPAL TENSILE STRAIN
SITE E

APPLIED FORCE

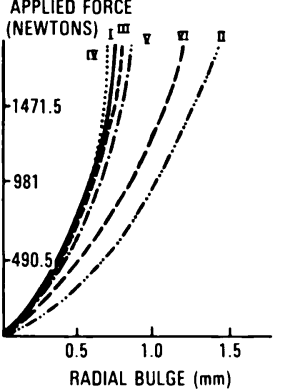

RADIAL BULGE $(\mathrm{mm})$

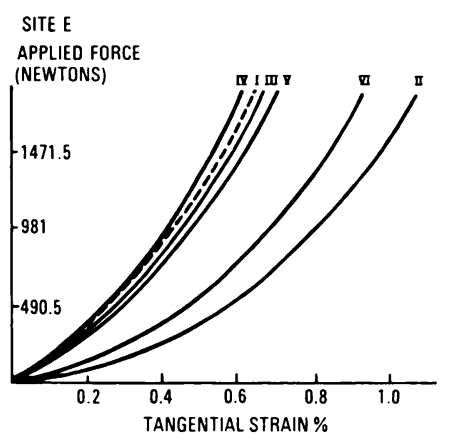

Fig. 5
The variation in strain/force and bulge/force patterns at sites 11 and $\mathrm{E}$ in the six specimens.

Anterior offset loading increased the tensile strain but decreased the compression strain at the bases of the pedicles, while both compression and tensile strains were reduced in the pars interarticularis. The greatest bulge was at the anterior surface of the disc, while tangential strain was maximal at the posterior surface.

\section{DISCUSSION}

From our findings it is not possible to determine the absolute stresses on the surfaces of L4 or of the L4-5 disc because their elastic constants cannot be independently determined, their values may be altered by the post-mortem changes, and the experimental system of loading does not accurately reflect the conditions in life. Nevertheless, we believe these results give some indication of the relative distribution of surface strain in vivo.

The result of the rosette strain gauge analyses of L4 confirm and extend the findings of our previous photo-elastic and brittle coat experiments on the lumbar spine (Shah et al. 1976). It is apparent that strains are not uniformly distributed over the surface of the vertebra. In experiments on central axial loading the highest compression strains occur near the bases of the pedicles and on both the superficial and deep surfaces of the pars 


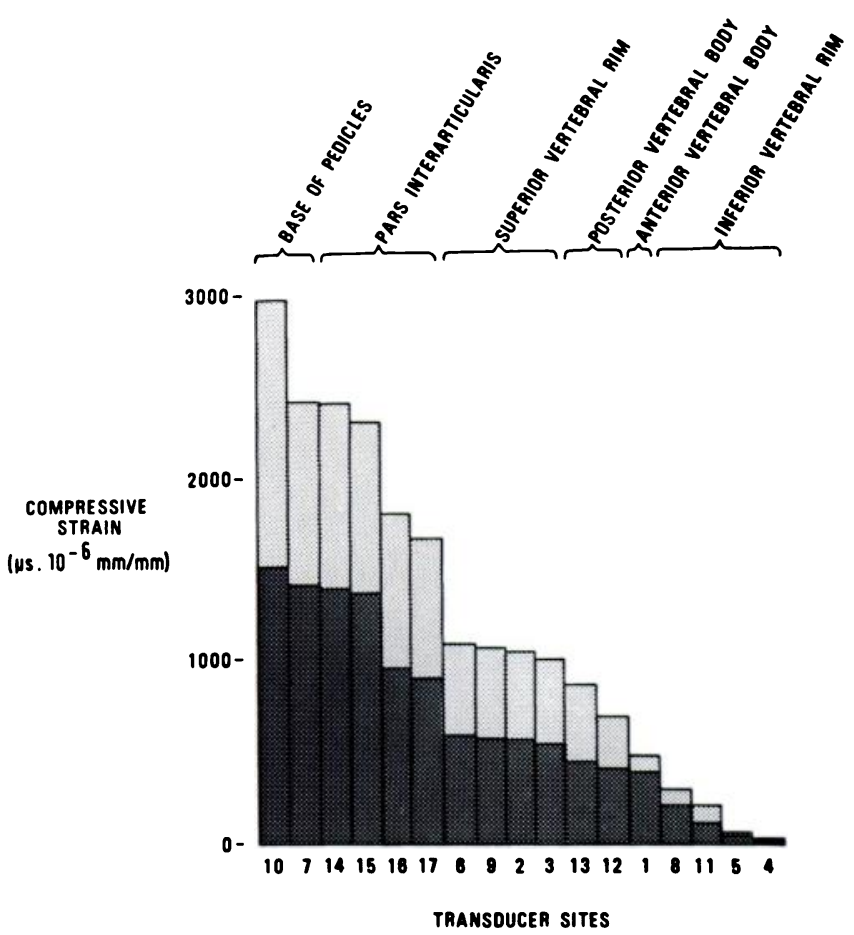

Fig. 6

Principal compressive strains on the surfaces of all six vertebrae in descending order, showing the ranges of values.

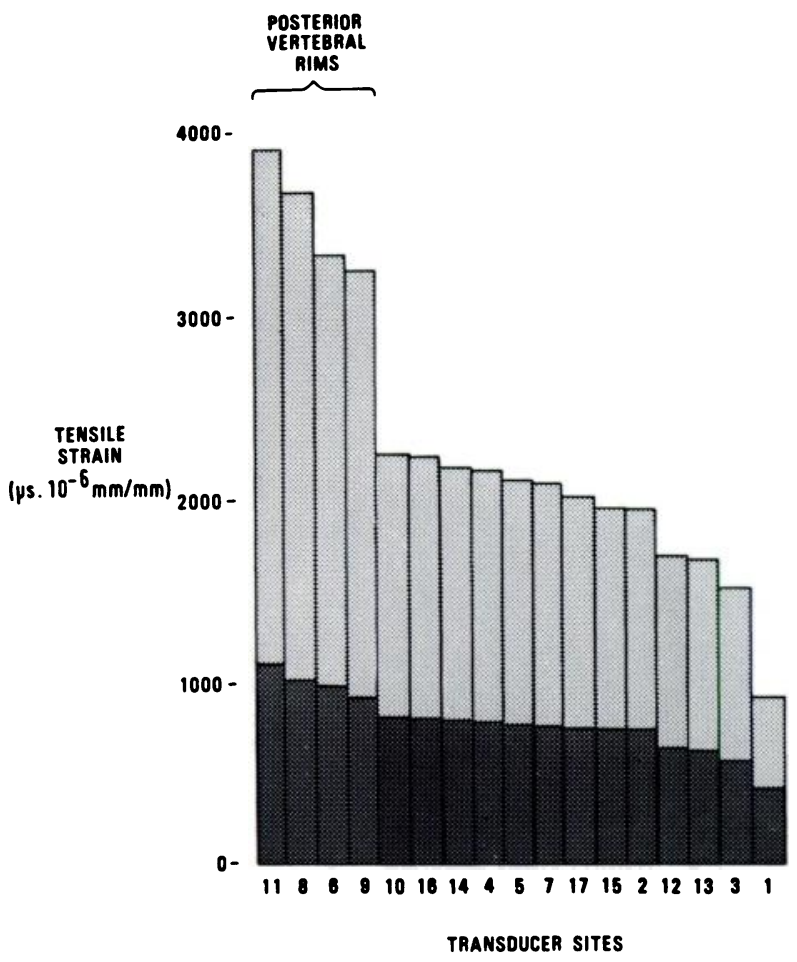

Fig. 7

Principal tensile strains on the surfaces of all six vertebrae in descending order, showing the ranges of values. interarticularis. These strains are further increased by posterior offset loading simulating extension. The findings indicate that the posterior elements of the spine transmit a substantial part of the load, and that this is increased by hyperextension. It is likely that stress fractures leading to spondylolisthesis are produced by repeated extension under load, as suggested by Cyron, Hutton and Troup (1976), rather than by flexion, as suggested by Stewart (1953).

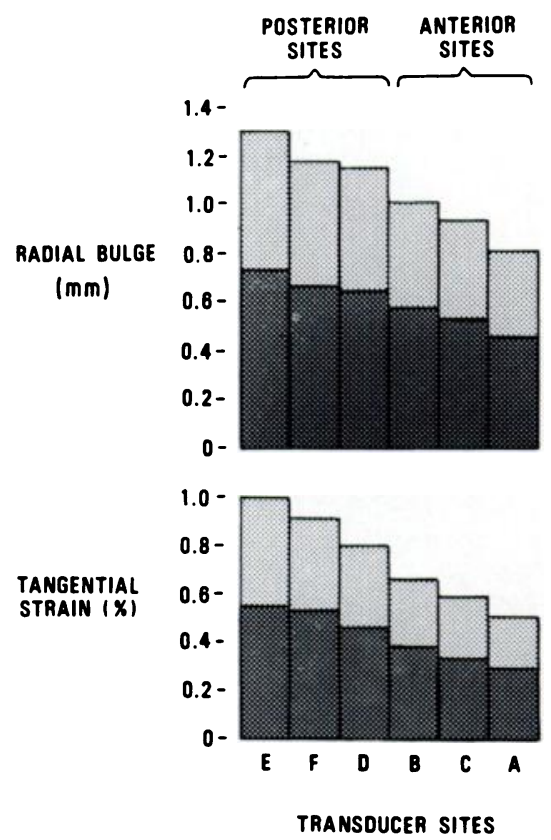

Fig. 8

Radial bulges and tangential strains on the surfaces of all six discs in descending order, showing the ranges of values.

\begin{tabular}{|c|c|c|c|c|c|c|c|c|c|c|c|c|c|}
\hline \multicolumn{7}{|c|}{ VERTEBral BoOY } & \multicolumn{7}{|c|}{ INTERVERTEBRAL DISC } \\
\hline \multicolumn{3}{|c|}{$\begin{array}{l}\text { PRIMCIPAL } \\
\text { COMPRESSION }\end{array}$} & \multirow{2}{*}{ SITES } & \multicolumn{3}{|c|}{$\begin{array}{l}\text { PRINCIPAL } \\
\text { TENSION }\end{array}$} & \multicolumn{3}{|c|}{$\begin{array}{l}\text { TANGEMTIAL } \\
\text { STRAIM }\end{array}$} & \multirow{3}{*}{ SITES } & \multicolumn{3}{|c|}{$\begin{array}{l}\text { RADIALL } \\
\text { BULGE }\end{array}$} \\
\hline AO & 6 & PO & & AO & c & PO & AO & c & PO & & AO & c & PO \\
\hline & & & $\begin{array}{l}\text { PARS } \\
\text { INTERARTICULARIS }\end{array}$ & & & & & & & & & & \\
\hline & & & $\begin{array}{l}\text { POST. VERT RIM } \\
\text { \& PEOICLE }\end{array}$ & & & & & & & POST. DISC WALL & & & \\
\hline & & & ANT. VERT. RIM & & & & & & & ANT. DISC WALL & & & \\
\hline
\end{tabular}

Fig. 9

Summary of effects of anterior offset (AO) and posterior offset (PO) loads compared with a central (C) load.

With central axial load, the highest tensile strains occurred on the posterolateral aspects of the vertebral rims. The significance of this finding will be discussed later.

Our investigation of the L4-5 disc extends work by Virgin (1951), Hirsch (1955), Brown, Hansen and Yorra (1957), Markolf (1972), and Markolf and Morris (1974), who all tested isolated segments of the spinal column under load and measured the expansion of the 
disc. We have confirmed that the bulge of the disc increases non-linearly under load, but in addition we have shown that tangential strain of the disc wall behaves in a similar manner. We have shown that on central axial load, both the tangential strain and the radial bulge are maximal at the posterolateral surfaces of the disc. This finding is consistent with the disc stresses computed by Kraus (1973), and with the findings of Farfan (1973) and Jayson and Barks (1973), who showed that degenerative changes usually start in the posterolateral and posterior aspects of the disc wall.
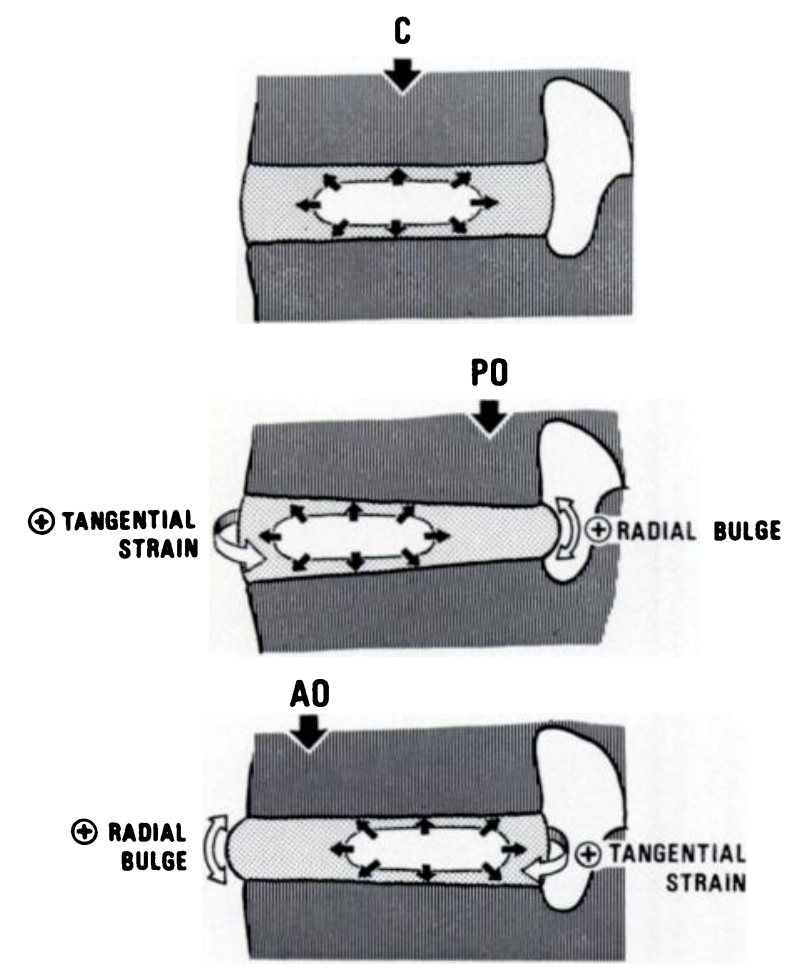

Model of Disc

Fig. 10

Suggested nuclear movement produced by anterior offset (AO) and posterior offset (PO) loads.

The strain distribution observed in central axial loading can be interpreted in terms of the hydrostatic model of the intervertebral disc proposed by Schmorl (1927). In this model the nucleus is assumed to be hydrostatic and isotropic, so that under axial compression it exerts uniform pressure in all directions on the interior surface of the annulus. The fibres of the annulus consequently develop tensile strains which are transmitted to the vertebral rim to which they are attached. This would explain the high tensile strains recorded on the rims of the fourth lumbar vertebra.

The fact that disc bulge and tangential strain, together with tensile strain on the vertebral rim surface, are maximal at the posterior aspects of the disc wall and vertebral rim could be accounted for by the nucleus being slightly offset posteriorly and by the shape of the disc in transverse section. Anisotropy of the elastic constants of the annulus could also give the same results. However, both hypotheses suggest that, at any site, offset loading would increase or decrease disc bulge and tangential strain together, which is contrary to our results. However, these findings can be accounted for if the nucleus is capable of displacement, as indicated in Figure 10. We suggest that an offset compressive force, roughly corresponding to that in flexion and extension, pushes the nucleus in the opposite direction and accounts for the high tangential strain in the parts of the annulus away from the offset load, with a radial bulge on the same side. This possibility was suggested by Steindler (1935) but without experimental proof.

We have undertaken preliminary experimental observations to detect such nuclear movements by observing the position of the nucleus pulposus on discography in three further post-mortem specimens with anterior and posterior offset loading (Fig. 11). Displacement of the nuclear cavity was seen. It seems likely that such nuclear displacement can occur during flexion and extension in life.

With ageing and degeneration of discs the nucleus becomes more fibrous (Happey et al. 1969), with increased interaction of proteoglycans with collagen (Adams and Muir 1976). This may limit the normal movement of the nucleus, and the hydrostatic pressures
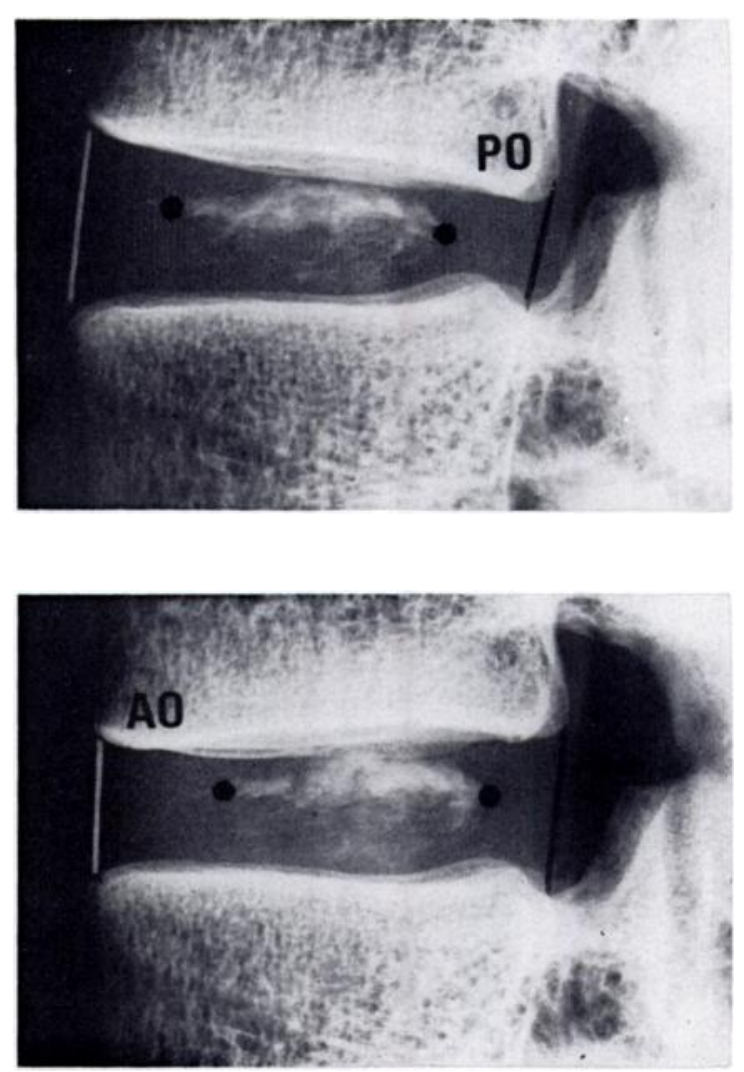

Fig. 11

Nuclear movement with anterior offset (AO) and posterior offset (PO) loads seen by discography. 
may be redistributed in an anisotropic fashion with localised sites of high stresses at the inner wall of the annulus. Further research is necessary to determine the altered distribution of strain in both bone and disc surfaces in degenerate spines. Biochemical changes could lead to alteration in the biochemical properties of the spine and exacerbate spinal damage. In turn, such altered distributions of stress may themselves initiate and potentiate further changes in the biochemical structure of the collagen and proteoglycan of the intervertebral disc, so that a vicious circle develops with progressive spinal damage.

This work was performed with the assistance of a grant from the Arthritis and Rheumatism Council for Research, to whom we are most grateful.

\section{REFERENCES}

Adams, P., and Muir, H. (1976) Qualitative changes with age of proteoglycans of human lumbar discs. Annals of the Rheumatic Diseases, 35, 289-296.

Brown, T., Hansen, R. J., and Yorra, A. J. (1957) Some mechanical tests on the lumbosacral spine with particular reference to the intervertebral discs. Journal of Bone and Joint Surgery, 39-A, 1135-1164.

Cyron, B. M., Hutton, W. C., and Troup, J. D. G. (1976) Spondylolytic fractures. Journal of Bone and Joint Surgery, 58-B, 462-466.

Dally, J. W., and Riley, W. F. (1965) Experimental Stress Analysis. New York: McGraw-Hill Book Co. Inc.

Farfan, H. F. (1973) Mechanical Disorders of the Low Back. Philadelphia: Lea and Febiger.

Happey, F., Pearson, C. H., Naylor, A., and Turner, R. L. (1969) The ageing of the human intervertebral disc. Gerontologia, 15, $174-188$.

Hirsch, C. (1955) The reaction of intervertebral discs to compression forces. Journal of Bone and Joint Surgery, 37-A, 1188-1196.

Jayson, M. I. V., and Barks, J. S. (1973) Structural changes in the intervertebral discs. Annals of the Rheumatic Diseases, 32, 10-15.

Kraus, H. (1973) Stress analysis. In Mechanical Disorders of the Low Back, pp 111-133. Edited by H. F. Farfan. Philadelphia: Lea and Febiger.

Lanyon, L. E. (1974) Experimental support for the trajectorial theory of bone structure. Journal of Bone and Joint Surgery, 56-B, 160-166.

Markolf, K. (1972) Deformation of the thoracolumbar intervertebral joints in response to external loads. Journal of Bone and Joint Surgery. 54-A, 511-533.

Markolf, K. L., and Morris, J. M. (1974) The structural components of the intervertebral disc. Journal of Bone and Joint Surgery, 56-A, 675-687.

Schmorl, G. (1927) Die pathologische Anatomie der Wirbersäule. Verhandlungen den Deutschen Orthopädischen Gesellschafi, $21,3-41$.

Shah, J. S., Coggins, J., Rogers, R., Jayson, M. I. V., and Hampson, W. G. J. (1976) Surface strain distribution in isolated single lumbar vertebrae. Annals of the Rheumatic Diseases, 35, 51-55.

Stewart, T. D. (1953) The age incidence of neural-arch defects in Alaskan natives, considered from the standpoint of etiology. Journal of Bone and Joint Surgery, 35-A, 937-950.

Steindler, A. (1935) Mechanics of Normal and Pathological Locomotion in Man. Springfield, Illinois: Charles C. Thomas.

Virgin, W. J. (1951) Experimental investigations into the physical properties of the intervertebral disc. Journal of Bone and Joint Surgery, 33-B, 607-611 